Problemgeschichte der Dialektik in der Neuzeit I 


\author{
Hans Heinz Holz
}

\title{
EINHEIT UND WIDERSPRUCH
}

\author{
Problemgeschichte der Dialektik \\ in der Neuzeit
}

I

Die Signatur der Neuzeit

Verlag J. B. Metzler

Stuttgart $\cdot$ Weimar 
Die Deutsche Bibliothek - CIP-Einheitsaufnahme

Holz, Hans Heinz:

Einheit und Widerspruch : Problemgeschichte der Dialektik in der Neuzeit / Hans Heinz Holz. - Stuttgart ; Weimar : Metzler

ISBN 978-3-476-01558-7

Bd. 1. Die Signatur der Neuzeit. - 1997

ISBN 978-3-476-01555-6

ISBN 978-3-476-03706-0 (eBook)

DOI 10.1007/978-3-476-03706-0

ISBN 978-3-476-01558-7 (Gesamtwerk)

ISBN 978-3-476-01555-6 (Band I)

Dieses Werk einschließlich aller seiner Teile ist urheberrechtlich geschützt. Jede Verwertung außerhalb der engen Grenzen des Urheberrechtsgesetzes ist ohne Zustimmung des Verlages unzulässig und strafbar. Das gilt insbesondere für Vervielfältigungen, Übersetzungen, Mikroverfilmungen und die Einspeicherung und Verarbeitung in elektronischen Systemen.

(C) 1997 Springer-Verlag GmbH Deutschland

Ursprünglich erschienen bei J. B. Metzlersche Verlagsbuchhandlung und Carl Poeschel Verlag GmbH in Stuttgart 1997 
Für Silvia 


\section{Inhalt}

Seite

$\mathrm{X}$ Abkürzungen

XIII Vorwort

1 Einleitung

1 1. Problemfelder der Dialektik

12 2. Idealistische und materialistische Dialektik

$20 \quad 3$. Systematisierung der Dialektik

39 I. Hauptstück: Der Anfang der Neuzeit

41 1. Kapitel: Die historische Ausgangslage

41 1.Der Aufgang der Neuzeit

46 2. Epochenschwelle

56 3. Rahmenbedingungen des Epochenwandels

65 4. Die neue Wissenschaftsgesinnung

70 2. Kapitel: Die Übergangszeit der Renaissance

70 1. Das Profil der Renaissance

76 2. Politische und ökonomische Voraussetzungen

81 3. Die zwiefältige Linie der Renaissance-Philosophie

89 3. Kapitel: Die Unendlichkeit der Welt

89 1. Die "grosse Wende«

94 2. Giordano Bruno

101 3. Kepler

105 4. Kapitel: Galilei

105 1. Experiment und Konstruktion

2. Ontologische und mathematische Interpretation

114 der Wirklichkeit

119 3. Geometrie des Raumes und mechanistisches Weltbild

129 II. Hauptstück: Descartes

131 1. Kapitel: Die Selbstbegründung des Denkens

131 1. Von der natürlichen Theologie zur Ontologie

138 2. Das natürliche Licht

148 3. Der universelle Zweifel und die Ich-Gewissheit 
2. Kapitel: Die Restitution der Weltgewissheit 1. Vom Cogito zur res cogitans

165 2. Die Konstruktion Gottes

3. Wahrheit und Irrtum

3. Kapitel: Die Struktur der Welt

1. Die res extensa

2. Die Geometrisierung der Welt

196 4. Kapitel: Descartes und die Folgen

205 III. Hauptstück: Antworten auf Descartes

207 1. Kapitel: Malebranche

217 2. Kapitel: Hobbes

222 3. Kapitel: Spinoza - die Wende der cartesischen Wende

222 1. Über Descartes hinaus

2. Ontologie als radikale Gotteslehre: der »Ethik» erster Teil

3. Spinozas Eleatismus - die Wirklichkeit als blosses Phänomen

261 IV. Hauptstück: Leibniz

1. Kapitel: Die Auseinandersetzung mit dem Cartesianismus

1. Die Bedeutung des Descartes im 17. Jahrhundert

2. Leibniz und Malebranche

3. Die »Bemerkungen zum Allgemeinen Teil der cartesischen Prinzipien“

4. Leibniz und Spinoza

2. Kapitel: Gott, Vollkommenheit und Welt im ersten System-Entwurf

1. Der Horizont des ersten System-Entwurfs

2. Gott als Infinitesimal

3. Das Optimum in der Natur

4. Das moralische Optimum

5. Erste Ursache und notwendig Seiendes

324 7. Die Dialektik des Gottesbegriffs 
334

334

339

346

354

362

371

378

378

381

386

390

396

396

402

407

421

425

431

441

448

455 Anmerkungen

$545 \quad$ Namenregister Kräfte

8. Schluss

3. Kapitel: Der neue Substanzbegriff

1. Das Eine und das Viele

2. Die substantiellen Formen

3. Das Ausdrücken

4. Praedicatum inest subiecto

5. Die Materialität der Welt

6. Gott, Naturgesetz, Vernunft

4. Kapitel: Die beste aller möglichen Welten

1. Theodizee oder die Rechtfertigung Gottes

2. Mögliche Welten

3. Möglichkeit und Wirklichkeit

4. Vollkommenheit

5. Kapitel: Das monadologische Weltmodell

1. Die Rationalität des Faktischen

2. Der universelle Zusammenhang

3. Das notwendig Seiende

4. Die Wechselwirkung individueller Substanzen

5. Ursprüngliche und abgeleitete, aktive und passive

6. Der Begriff der Monade

7. Die »wohlbegründete Erscheinung" 


\section{Abkürzungen}

In den Anmerkungen zu diesem Band werden durchgängig die folgenden Abkürzungen gebraucht:

Descartes, A-T $=$ Oeuvres de Descartes, publiés par Charles Adam et Paul Tannery, nouvelle édition Paris 1996. Bandzahl in römischer, Seitenzahl in arabischer Ziffer.

Hegel, GW = Georg Wilhelm Friedrich Hegel, Gesammelte Werke, kritische Edition der Rheinisch-Westfälischen Akademie der Wissenschaften, Düsseldorf.

Hegel, W = Georg Wilhelm Friedrich Hegel, Werke in 20 Bänden (Theorie-Werkausgabe des Suhrkamp Verlags), Frankfurt am Main $1970 \mathrm{ff}$.

Die Theorie-Werkausgabe wurde immer da benutzt, wo die betreffenden Bände der Gesammelten Werke bei Abfassung des Manuskripts noch nicht vorlagen. Wo die Gesammelten Werke zitiert werden, ist die entsprechende Stelle in der Theorie-Werkausgabe ebenfalls nachgewiesen, um den Gebrauch zu erleichtern. Enzyklopädie und Rechtsphilosophie werden nach Paragraphen zitiert, sodass sie mühelos in jeder Ausgabe auffindbar sind.

Leibniz, AA = Gottfried Wilhelm Leibniz, Sämtliche Schriften und Briefe, hg. von der Deutschen Akademie der Wissenschaften zu Berlin, Reihennummer und Bandzahl in römischen Ziffern, Seitenzahl in arabischen Ziffern.

Leibniz, $\mathrm{C}=\mathrm{G}$. W. Leibniz, Opuscules et fragments inédits, par Louis Couturat, Paris 1903. Neudruck Hildesheim 1966.

Leibniz, E = Gottfried Wilhelm Leibniz, Opera Philosophica Omnia, instruxit J. E. Erdmann, Berlin 1840. Neudruck Aalen 1959.

Leibniz,

Gerh/Phil = G. W. Leibniz, Die philosophischen Schriften, hg. von C. J. Gerhardt, Berlin 1875. Neudruck Hildesheim 1965. Bandzahl in römischen, Seitenzahl in arabischen Ziffern. 
Leibniz, Grua = G. W. Leibniz, Textes inédits, publiés et annotés par Gaston Grua, Paris 1948

Leibniz, KS = Gottfried Wilhelm Leibniz, Kleine Schriften zur Metaphysik, hg. und übers. von Hans Heinz Holz, Darmstadt 1965.

Leibniz, Lewis = Lettres de Leibniz a Arnauld, par Geneviève Lewis, Paris 1952.

Leibniz, NE = Gottfried Wilhelm Leibniz, Neue Abhandlungen über den menschlichen Verstand, hg. und übers. von Wolf von Engelhardt und Hans Heinz Holz, 2 Bände, Darmstadt 1959.

Leibniz, Theod. $=$ Herrn Gottfried Wilhelms Freiherrn von Leibniz Theodicee, hg. von Johann Christoph Gottsched, Leipzig 1744. Neuausgabe von Hubert Horstmann, Berlin 1996.

Lenin, LW = Wladimir Iljitsch Lenin, Werke, ins Deutsche nach der vierten russischen Ausgabe übertragen und vom Institut für Marxismus-Leninismus beim Zentralkomitee der SED herausgegeben.

Karl Marx/

Friedrich Engels,

MEW = Karl Marx / Friedrich Engels, Werke, herausgegeben vom Institut für Marxismus-Leninismus beim Zentralkomitee der SED, Berlin.

In den verschiedenen Ausgaben älterer Autoren - insbesondere bei Leibniz - wird die Schreibweise auf verschiedene Weise wiedergegeben: einmal original getreu, ein andermal modernisiert. Wir folgen jeweils der Schreibweise der zitierten Ausgabe, ohne diese zu homogenisieren.

Weitere Abkürzungen, von denen nur in einzelnen Kapiteln Gebrauch gemacht wird, werden jeweils beim ersten Zitat in dem entsprechenden Kapitel angegeben. Die Anmerkungen sind für jedes Kapitel gesondert numeriert, entsprechend werden in jedem Kapitel beim ersten Zitieren die bibliographischen Angaben aufs neue voll angegeben. 


\section{Vorwort}

Die vorliegende Darstellung unterfängt sich nicht, die Geschichte der Dialektik seit Beginn der Neuzeit zu entfalten. Dazu wäre ein viel grösserer Raum nötig, denn diese Geschichte verzweigt sich in zahlreiche Arme und Seitentriebe, die wohl oft auf den Hauptstrom zurückgewirkt haben, aber doch den Gang der Problementwicklung nicht wirklich zu beeinflussen vermochten. Hier weitere Klarheit zu schaffen, muss Einzelstudien überlassen bleiben. ${ }^{1}$

Was in dieser Problemgeschichte versucht wird, ist die Herausarbeitung von Knotenpunkten im Fortgang des philosophischen Denkens - Knotenpunkte, an denen die Probleme sich systematisch verdichten und weiterweisende Lösungsansätze vorgeschlagen werden, die zu neuen Systemstrukturen führen. Dabei wird vorausgesetzt, dass die Veränderung philosophischer Denkmuster sich in einem Traditionskontinuum vollzieht, dass also die Philosophie - wie sehr sie auch mit den gesellschaftlichen und wissenschaftlichen Entwicklungen verbunden und von ihnen abhängig ist - durchaus eine eigene Geschichte hat, die durch den Charakter philosophischer Probleme und ihrer begrifflichen Formulierung geprägt ist. ${ }^{2}$

Doch noch in einem weiteren Sinne verfährt die vorliegende Darstellung restriktiv. Kürzlich hat an einem Symposion ${ }^{3}$ Nicola de Domenico nachdrücklich die These verfochten, dass es "zwei Dialektiken" gäbe - eine des common sense und der Alltagserfahrung und eine spekulative, die das Ganze von Welt und die ihm angemessene Erkenntnisart im Blick hat. Diese Unterscheidung ist sinnvoll; sie lässt sich auch so ausdrücken, dass es eine Dialektik im Felde der Verstandesbestimmungen gibt (wozu auch die der Wissenschaften gehört), und eine Dialektik der Vernunft (ihrer Tätigkeit und Gegenstände). Ich teile die Auffassung von de Domenico, dass eine Grundlegung der Verstandesdialektik nur durch die spekulative Vernunft geleistet werden kann.

Die folgende Darstellung beschränkt sich auf diesen Bereich der spekulativen Dialektik. Selbstverständlich gibt es auch eine Dialektik unmittelbar im gesellschaftlichen Leben (und sie ist sogar der Anlass zu dialektischer Reflexion überhaupt). Deren theoretisches Bild hat die 
Geschichts- und Staatsphilosophie von Machiavelli über Hobbes und Rousseau bis zu Marx und Lenin gezeichnet. Die Kategorien aber, mit denen diese theoretischen Entwürfe arbeiten, haben ihren Ursprung und ihre Legitimation in spekulativen Konzepten, was da deutlich wird, wo diese Kategorien selbst Gegenstand der Reflexion werden, wie zum Beispiel bei Kant und Hegel. Erst wenn wir den Gang spekulativen Denkens $^{4}$ nachvollziehen, werden die Theorien, die es mit der Dialektik der Faktischen zu tun haben, ihre zureichende Begründung erhalten.

Dass dem so ist, muss die Darstellung selbst einsichtig machen. Der Weg der spekulativen Dialektik entlang der Knotenlinie ${ }^{5}$ ihrer Probleme hat damit nicht nur eine historiographische, sondern ebensosehr eine systematische Bedeutung. Dialektik ist, was sie ist, nur in der Einheit ihrer Geschichte. Sie ist so sehr Geschichte, dass ihre Identität sich zuweilen in die Alteritas ihrer gewordenen Gestalten aufzulösen scheint. Auf die Frage, was Dialektik sei, ist darum nicht mit einer einfachen Definition zu antworten. Dennoch ist sie nicht nur Geschichte, sondern auch deren systematischer Begriff. Der kann zwar nicht am Anfang gegeben werden, aber ein Vorbegriff 6 ihrer Probleme und Aporien ist unerlässlich, damit die Einheit der Denkbewegung von der geschichtlichen Vielfalt nicht verdeckt bleibt; nur darf der Vorbegriff nicht den Zugang zu dem erst noch zu gewinnenden Begriff versperren.

Dass diese Darstellung mit der Neuzeit beginnt, hat einen guten Grund. Die neuzeitliche Rückwendung auf das Selbstbewusstsein hat der Philosophie insgesamt eine neue Dimension gegeben. Durch sie erhalten die Formbestimmungen der ontologischen Dialektik der Antike erst eine Reflexionsebene, von der aus sie begründet und nicht nur an sich selbst sichtbar gemacht und beschrieben werden können. Allerdings macht die Begründung der Dialektik in einem Reflexionsverhältnis, dessen Referenzpunkt das Ich ist, diese zu einer midealistischen ${ }^{7}{ }^{7}$ Wie die darin liegende weltanschauliche Relativität aufzulösen sei, ist selbst ein Problem der Dialektik (und ein dialektisches Problem), das erst im Gang des spekulativen Denkens an geschichtlich bestimmbarem Ort erkennbar wird.

Eine Problemgeschichte, der es um die ausformulierte Problemgestalt und ihre systematische Verarbeitung geht, wird innerhalb des historischen Verlaufs Akzente zu setzen haben. Problemstellungen bereiten sich vor im Ringen um Antworten auf gesellschafts- und wissenschaftsgeschichtlich neue Situationen ${ }^{8}$, aber auch in dem Bemühen, Aporien traditioneller philosophischer Systematiken zu 
überwinden. Für die Formulierung der Dialektik in der Neuzeit ist der Cartesianismus die Herausforderung. Die von Galilei und Descartes vollzogene Verweltlichung der Wissenschaft und Philosophie machte es nötig, für die philosophischen Fragen nach dem Grund und nach dem Absoluten einen neuen Zugriff zu finden. Diese Aufgabe bestimmt in der einen oder anderen Weise das Philosophieren im 17. und 18. Jahrhundert. Leibniz reagierte darauf mit der Ausarbeitung dialektischer Konzepte. Er stellt den ersten Höhepunkt in der Geschichte der neuzeitlichen Dialektik dar. Kant, der dann unter Aufnahme der Impulse des englischen Empirismus die cartesische cogito-Philosophie zu einem transzendentalen Subjektivismus radikalisierte, gab den Anstoss zum zweiten grossen Ansatz einer dialektischen Konstruktion der klassischen metaphysischen Probleme durch Hegel. Aus dieser Charakterisierung der Problementwicklung ergibt sich, dass die Hauptstücke über Leibniz und Hegel zu tragenden Pfeilern im Gesamtaufbau werden. ${ }^{9}$ Jede Weiterentwicklung der Dialektik wird von den kategorialen Strukturen auszugehen haben, die in diesen beiden Weltmodellen ausgearbeitet wurden; hinter sie kann nicht mehr zurückgefallen werden - so wenig wie die Logik je hinter das Organon des Aristoteles zurückfallen konnte. Aus diesem Grund findet die Dialektik in ihrer Problemgeschichte die Elemente ihrer Systematik. ${ }^{10}$

Es ist ein Spezifikum der Dialektik, dass in ihr systematische und historische Aspekte ineinander übergehen und verschränkt sind. Zwar gilt das in gewissem Masse für Philosophie überhaupt, die nur um den Preis einer bornierten Verkürzung ihrer Problemgehalte sich der kritischen Rezeption ihrer Manifestationen in der Geschichte des Denkens entschlagen könnte. (Warum das so ist, lässt sich allerdings nur in einer Erörterung der Dialektik von Historizität und Präsenz dessen, was wir Kultur nennen, begründen und ist also selbst ein Problem der Dialektik). In besonderer Weise wird daher die kategoriale Verfassung dialektischer Philosophie durch die geschichtliche Situation mitdefiniert, in der sie entsteht; und sie verfällt dennoch nicht der Obsoleszenz des Vergangenen, das wir hinter uns gelassen haben, sondern behält als mögliche Formbestimmtheit des Denkens ihre Geltung. Dieser Status von Apriorität, der ins historisch Kontingente eingesenkt ist, macht die ganze Problemgeschicbte der Dialektik zu einem unverzichtbaren Bestandteil ibrer aktuellen Systematik. So ist es unvermeidbar, dass in ihrer Darstellung dem in die Geschichte Blickenden die Systemperspektive aufscheint, wie sie sich ihm gegenwärtig zeigt; nur eine antiquarisch verfahrende Geschichtsschreibung ${ }^{11}$ darf ihn dessenthalben tadeln. 


\section{Vorwort}

Zum Schluss dieses Vorworts habe ich zu danken: der Universität Groningen, an der eine achtzehnjährige Lehr- und Forschungstätigkeit mir den Spielraum gegeben hat, ein Werk wie das vorliegende (das auf die Epochen des Altertums und Mittelalters auszudehnen ich die Absicht habe) zustande zu bringen; meiner Frau Silvia, die bei der Verifizierung und Textherstellung des umfangreichen Apparats von Quellenzitaten und beim mühevollen Lesen der Korrekturen wesentlich geholfen hat; Frau Laurence Charpentier, die das Namensregister anfertigte; und nicht zuletzt dem Verlag, der dieses Werk in seine Obhut genommen und sein Erscheinen ermöglicht hat. 